\title{
Combination therapy with oral ursodeoxycholic and chenodeoxycholic acids: pretreatment computed tomography of the gall bladder improves gall stone dissolution efficacy
}

\author{
J R F Walters, K A Hood, D Gleeson, J P M Ellul, A Keightley, G M Murphy, R H Dowling
}

\begin{abstract}
In a five year study, 55 patients with radiolucent gall stones were treated with the combination of $7.5 \mathrm{mg}$ chenodeoxycholic acid (CDCA) and $5.0 \mathrm{mg}$ ursodeoxycholic acid (UDCA)/kg/day that is, half the monotherapeutic doses. Side effects were few but four patients could not tolerate the prescribed bile acids because of diarrhoea or nausea. Analysis of fasting duodenal bile confirmed that CDCA+UDCA converted supersaturated into unsaturated bile but the saturation indices did not predict the dissolution response. By actuarial analysis, the confirmed (by ultrasound $\times 2$ ) complete gall stone dissolution rates in all 55 patients were mean (SEM) $29(7) \%$ at 12 and $44(8) \%$ at 24 months. The advent of routine computed tomography before treatment enabled comparison of dissolution efficacy in those screened by computed tomography $(n=24)$, whose maximum gall stone attenuation was $<100$ Hounsfield units, with that in those not screened $(n=29)$. Although stone size and number were comparable, patients screened by computed tomography had significantly better dissolution rates $(p<0.025)$ than those not screened in this way. At 12 months, partial or complete gall stone dissolution rates were $93(7) \%$ in the screened and $55(11 \%)$ in the nonscreened patients. At 18 months, complete dissolution rates were $64(12 \%)$ and $20(9) \%$ respectively. Computed tomography before treatment is cost effective in selecting those patients likely to achieve gall stone dissolution on treatment with UDCA + CDCA.
\end{abstract}

Monotherapy with oral chenodeoxycholic acid (CDCA) or ursodeoxycholic acid (UDCA) is moderately effective in dissolving cholesterol rich gall stones. ${ }^{1-4}$ The dose related side effects of diarrhoea, hypertransaminasaemia, ${ }^{5}$ and hypercholesterolaemia, ${ }^{6}$ however, have limited the acceptability of CDCA as a gall stone dissolving agent. UDCA causes minimal side effects but in our experience its efficacy in dissolving gall stones completely is less than that of CDCA, partly because of secondary gall stone calcification. ${ }^{4}$ Furthermore, although less UDCA than CDCA is required to desaturate bile $^{7-9}$ and dissolve gall stones, the cost of monotherapy with UDCA is somewhat greater than that of CDCA. For these reasons, several groups $^{10-16}$ have advocated the combination of CDCA and UDCA, each at half their monotherapeutic doses, with the aim of maintaining dissolution efficacy while minimising side effects. We began using CDCA plus UDCA for gall stone dissolution in 1984 and report here the results of a five year study with this combination in 55 patients.

We, ${ }^{17}$ and others, ${ }^{18} 19$ have recently shown that no less than $50 \%$ of gall bladder stones which are lucent by conventional radiology (plain $x$ ray with or without oral cholecystography), look dense on computed tomography. These stones have high Hounsfield unit (HU) attenuation values and contain calcium in amounts that render complete dissolution with oral therapy unlikely. Indeed, over the past three years we have routinely screened all patients being considered for non-surgical management of their gall bladder stones by localised scanning of the gall bladder, and have accepted patients for treatment with oral CDCA+UDCA only if their stones had a maximum computed tomographic attenuation of $<100 \mathrm{HU}$. We show here that patients selected in this way have significantly higher partial and complete gall stone dissolution rates than those not so screened and suggest that routine computed tomography of the gall bladder before treatment is cost effective in predicting the dissolution response to oral bile acid therapy.

\section{Methods}

\section{PATIENT SELECTION}

\section{Admission criteria}

Fifty five patients were entered into the study between June 1984 and June 1989: their clinical details and gall stone characteristics are given in Table I. Eight patients had previously been treated with oral bile acids and were given the combination of CDCA plus UDCA for management of either recurrent $(n=2)$ or incompletely dissolved $(n=6)$ stones.

All 55 patients had specific, gall stone related symptoms (biliary colic) and all chose oral bile acids in preference to surgery or other nonsurgical management options. ${ }^{20}$ The presence of gall bladder stones was usually diagnosed first by ultrasonography. In addition, however, all patients underwent oral cholecystography which included a preliminary plain abdominal $x$ ray and, during the contrast study, erect and after fatty meal films. Liver function tests (serum albumin, bilirubin, alkaline phosphatase, and transaminase), serum lipids, and a full blood count were all normal before treatment. 
TABLE I Characteristics of patients treated with chenodeoxycholic acid plus ursodeoxycholic acid

\begin{tabular}{|c|c|c|c|}
\hline & $\begin{array}{l}\text { Overall } \\
\text { group }\end{array}$ & Not screened & $\begin{array}{l}\text { Screened by CT } \\
(<100 \mathrm{HU})\end{array}$ \\
\hline \multicolumn{4}{|l|}{ Year of entry to trial: } \\
\hline $1984-5$ & 10 & 10 & 0 \\
\hline $1985-6$ & 12 & 12 & 0 \\
\hline 1986-7 & 12 & 7 & $3^{\star}$ \\
\hline $1987-8$ & 9 & 0 & 9 \\
\hline $1988-9$ & 12 & 0 & 12 \\
\hline Total & 55 & 29 & $24^{\star}$ \\
\hline \multicolumn{4}{|l|}{ Patient characteristics: } \\
\hline $\begin{array}{l}\text { Age (yrs) mean (SEM) } \\
\text { (range) }\end{array}$ & $\begin{array}{c}50(2) \\
(23-91)\end{array}$ & $\begin{array}{c}49(3) \\
(23-91)\end{array}$ & $\begin{array}{c}52(3) \\
(28-81)\end{array}$ \\
\hline $\operatorname{Sex} \operatorname{ratio}(M: F)$ & $11: 44$ & $4: 25$ & $6: 18$ \\
\hline Previous bile acid therapy & 8 & 6 & $1^{\star}$ \\
\hline \multicolumn{4}{|l|}{ Stone characteristics: } \\
\hline Single & 13 & 7 & $5^{\star}$ \\
\hline Multiple & 42 & 22 & $19^{\star}$ \\
\hline Median number & 5 & & \\
\hline $\begin{array}{l}\text { Maximum diameter of largest stone } \\
(\text { mean }(\mathrm{SEM}))\end{array}$ & $8 \cdot 1(0 \cdot 6)$ & $8 \cdot 0(0 \cdot 9)$ & $8 \cdot 3(0 \cdot 8)$ \\
\hline
\end{tabular}

CT= computed tomography.

* Two further patients are not included in either subgroup. They were screened with pretreatment CT but were treated despite having CT values greater than $100 \mathrm{HU}$.

\section{Exclusion criteria}

In both sub groups (see below), patients were excluded from the study if calcification of the gall stones was seen by conventional radiology, if the gall bladder failed to opacify during oral cholecystography (two consecutive studies, the second with a double dose of contrast), or if the maximum diameter of the largest stone exceeded $20 \mathrm{~mm}$. Women of child bearing age were excluded from treatment unless they were taking effective contraceptive measures.

LOCALISED COMPUTED TOMOGRAPHY OF THE GALL BLADDER

Computed tomography of the gall bladder region was performed routinely in all gall stone patients being considered for non-surgical treatment from the end of 1986 onwards. Imaging was performed on a Phillips Tomoscan 350 at a setting of $120 \mathrm{kV}$ and $200 \mathrm{~mA}$ with a scan time of five seconds, and usually comprised five to seven $6 \mathrm{~mm}$ contiguous 'cuts' to scan the gall bladder region completely. Based on our previous findings, ${ }^{17}$ we excluded from treatment any patient whose stones had a maximum computed tomogram density in excess of $100 \mathrm{HU}$.

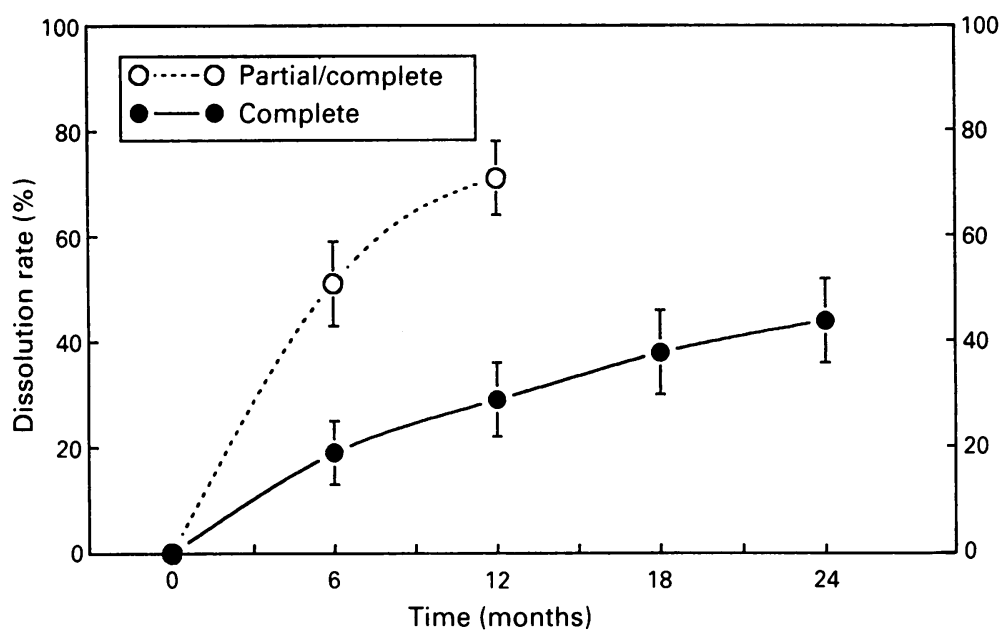

Figure 1: Gall stone dissolution rates (mean (SEM)) in the entire group of 55 patients calculated by life table analysis at six monthly intervals, showing complete gall stone dissolution and partial plus complete dissolution.
BILE LIPID ANALYSIS

Fasting, bile rich duodenal fluid was obtained by aspiration from 31 patients before treatment and from 22 after approximately six weeks of therapy. Total bile acid, phospholipid, and cholesterol concentrations were measured, and the biliary cholesterol saturation index (SI) determined, as previously described. ${ }^{2}$

\section{TREATMENT AND FOLLOW UP}

Patients were given the combination of approximately $7.5 \mathrm{mg} \mathrm{CDCA} / \mathrm{kg} /$ day and $5.0 \mathrm{mg}$ UDCA $/ \mathrm{kg} /$ day, which was taken as a single bedtime dose.

The patients were seen for follow up every two to three months, at which time compliance in taking the prescribed medication was checked, symptoms were assesed, and side effects were noted. Fasting serum lipids and liver function tests were also measured at each visit.

\section{ASSESSMENT OF GALL STONE DISSOLUTION}

RESPONSE

Gall stone dissolution was assessed every six months by ultrasonography. Partial gall stone dissolution was defined as a $50 \%$ decrease in gall stone number or volume, or both. Complete dissolution was confirmed by two consecutive ultrasonographic examinations, at least one month apart, during continued bile acid treatment. In this study, oral cholecystography was used to exclude acquired gall stone calcification or blockage of the cystic duct in patients who had not shown ultrasonographic evidence of gall stone dissolution after one year of therapy.

Treatment was withdrawn at different times for the following reasons: (i) at one year in compliant patients who had shown no radiological evidence of gall stone dissolution; (ii) after a total of two years treatment in those who had shown partial gall stone dissolution after one year but had not progressed to complete dissolution; (iii) at any time after confirmation of complete gall stone dissolution; or (iv) because of unacceptable side effects.

Gall stone dissolution rates were assessed by acturial or life table analysis (LTA). ${ }^{21}$ The results in patients whose treatment was withdrawn at one year because of non-response, were retained in the number 'at risk' for subsequent analysis. The results in patients who defaulted or withdrew from the trial because of complications of treatment, however, were censored from subsequent analysis. Except where otherwise indicated, the results are expressed as mean (SEM). Dissolution rates are compared by the log rank test or by the Student's $t$ test as appropriate.

\section{Results}

\section{PATIENT CHARACTERISTICS}

Patient characteristics and details of their gall stones are shown in Table I. Of the 55 patients who joined the study, 29 were accepted before computed tomography of the gall bladder was performed routinely and constituted one subgroup. The other subgroup was formed by 24 
TABLE II Outcome of treatment in 55 gall stone patients entered into the study of combined ursodeoxycholic acid plus chenodeoxycholic acid

\begin{tabular}{|c|c|c|c|c|c|c|c|c|c|c|c|c|}
\hline & \multicolumn{12}{|c|}{ Time interval (mths) } \\
\hline & \multicolumn{4}{|c|}{ Overall group } & \multicolumn{4}{|c|}{ Not screened } & \multicolumn{4}{|c|}{$C T<100 \mathrm{HU}$} \\
\hline & $0-6$ & -12 & -18 & -24 & $0-6$ & -12 & -18 & -24 & $0-6$ & -12 & -18 & -24 \\
\hline \multicolumn{13}{|l|}{ No of patients: } \\
\hline At start of period & $55^{\star}$ & 35 & 26 & 22 & 29 & 20 & 16 & 15 & 24 & 13 & 8 & 5 \\
\hline Lost to follow up & 5 & 4 & 0 & 0 & 3 & 3 & 0 & 0 & 2 & 1 & $\begin{array}{lll}\circ \\
0\end{array}$ & 0 \\
\hline Withdrawn & 7 & 1 & 1 & 1 & 4 & 0 & 0 & 0 & 3 & 1 & 1 & 1 \\
\hline Assessed at end of period & 43 & 30 & $25 t$ & $21+$ & 22 & 17 & $15 t$ & $15 \dagger$ & 19 & 11 & $7 \dagger$ & $4 \dagger$ \\
\hline Complete or partial dissolution & 22 & 7 & - & - & 7 & 4 & - & - & 15 & 2 & - & - \\
\hline Complete dissolution & 8 & 4 & 3 & 2 & 2 & 1 & 1 & 2 & 6 & 3 & 2 & 0 \\
\hline
\end{tabular}

^Includes two patients not included in either subgroup (see Table I).

†Number includes patients whose treatment was withdrawn at 12 months having shown no dissolution (eight in the unscreened group and two in the screened group).

patients, all of whom had undergone computed tomography of their gall bladder and had stones with a maximum density of $<100 \mathrm{HU}$. Not included in either subgroup were two other patients who underwent computed tomography and had $\mathrm{HU}$ values greater than 100 , but who began treatment before our computed tomography selection criteria became firmly established.

As Table I shows, the clinical and gall stone characteristics were comparable in both subgroups of patients. Of the eight who had been treated with oral bile acids previously, two (one in each subgroup) had recurrent stones after complete stone dissolution wth CDCA or UDCA alone, three had had partial stone dissolution on UDCA, and three had failed to respond to ursocholic acid. ${ }^{22}$

\section{CLINICAL RESPONSE}

\section{Default/withdrawal}

Table II summarises the outcome of treatment in all 55 patients. A total of 19 patients did not complete the study - 10 were withdrawn and a further nine defaulted from follow up. Three withdrew because of recurrent biliary pain and underwent elective cholecystectomy. Two others who defaulted are also known to have undergone surgery. Forty two (76\%) reported no further symptoms from their gall stones during bile acid therapy.

\section{Side effects and complications}

Four patients were withdrawn because they could not tolerate the oral bile acid treatment two because of diarrhoea (one of whom had colonic diverticular disease) and two because they claimed that the treatment increased their pre-existing nausea. Of the three other patients withdrawn from the study, one developed a spinal artery thrombosis and another had failing eyesight; neither complication was thought to be related to therapy. The third elected to have lithotripsy for his $18 \mathrm{~mm}$ stone when this treatment became available.

Diarrhoea or loose stools occurred in 14 patients $(25 \%)$. This was transient in seven (whose bile acid dose remained unchanged), resolved on reducing the dose of CDCA in five but, as indicated above, persisted in the remaining two. No patient developed raised serum transaminase values or hyperlipidaemia and none was found to have developed secondary gall stone calcification.

\section{Gall stone dissolution}

The gall stone dissolution rates, calculated by LTA, are shown for all 55 patients (those screened and those not screened by computed tomography) in Figure 1.

At six months, the pooled complete gall stone dissolution rate was $19(6) \%$ and the partial plus complete dissolution rate was $51(8) \%$. The corresponding figures at 12 months were $29(7) \%$ and $71(7) \%$ respectively. Because of the trial design, there are no data for partial dissolution rates beyond 12 months but the complete dissolution rates rose to $38(8) \%$ and $44(8) \%$ at 18 and 24 months respectively.

\section{Effect of computed tomography on outcome of therapy}

In the subgroup of 24 patients screened by computed tomography who had a maximum stone attenuation of $<100 \mathrm{HU}$, the partial plus complete gall stone dissolution results were significantly better (log rank test for all time periods: $p<0.025$ ) than those in the subgroup of 29 patients who had not been screened (Fig 2). By one year, the partial plus complete rate in screened patients with HU scores of $<100$ was 93 (7\%) compared with $55(11) \%$ in those who had not been screened.

Over all time points, complete gall stone dissolution rates were significantly better in the screened group $(\mathrm{p}<0.025)$. At 12 months, the complete dissolution rates were $50(12) \%$ in the screened and $14(8) \%$ in the non-screened patients (Fig 3). After eighteen months' therapy, $64(12) \%$ of the screened patients showed complete dissolution compared with only $20(9) \%$ of the unscreened patients $(p<0 \cdot 01)$. As shown in Table I, this improved dissolution rate was not due to any difference in the characteristics of the patients or their stones.

BILE LIPID ANALYSIS

Analysis of duodenal bile showed that before treatment the biliary cholesterol saturation index was $1.32(0 \cdot 11)$. During treatment, the mean saturation index fell significantly to $0.74(0.06)$ $(p<0.001)$. To assess whether a pretreatment 


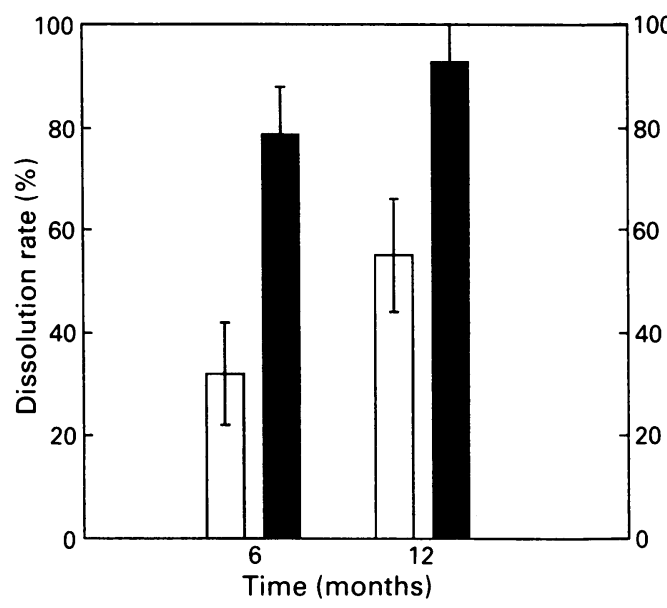

Figure 2: Partial plus complete gall stone dissolution rates (mean (SEM)) at six and 12 months calculated by life table analysis in the two subgroups: $(i) 24$ patients screened by computed tomography (solid boxes) and (ii) 29 patients not screened (open boxes).

saturation index of $<1.0$ could predict patients with non-cholesterol stones and thus a poor dissolution response, the outcome in the seven patients with a pretreatment index of $<1.0$ who completed one year's therapy was analysed. Only one of these achieved complete dissolution, two achieved partial dissolution, while the remaining four showed no radiological evidence of response. In contrast, of the 14 patients who had a pretreatment saturation index of $>1.0$ and who were treated for one year, five had complete dissolution, four had partial dissolution, and five had no response. However, the outcome in the two groups was not statistically different $(p>0 \cdot 3$ by Fischer's exact test). Furthermore, when saturation index results were compared in responding (partial or complete dissolution) and non-responding patients, there were no significant differences in results before or during treatment.

\section{Discussion}

These results confirm that the combination of oral UDCA plus CDCA effectively dissolves gall

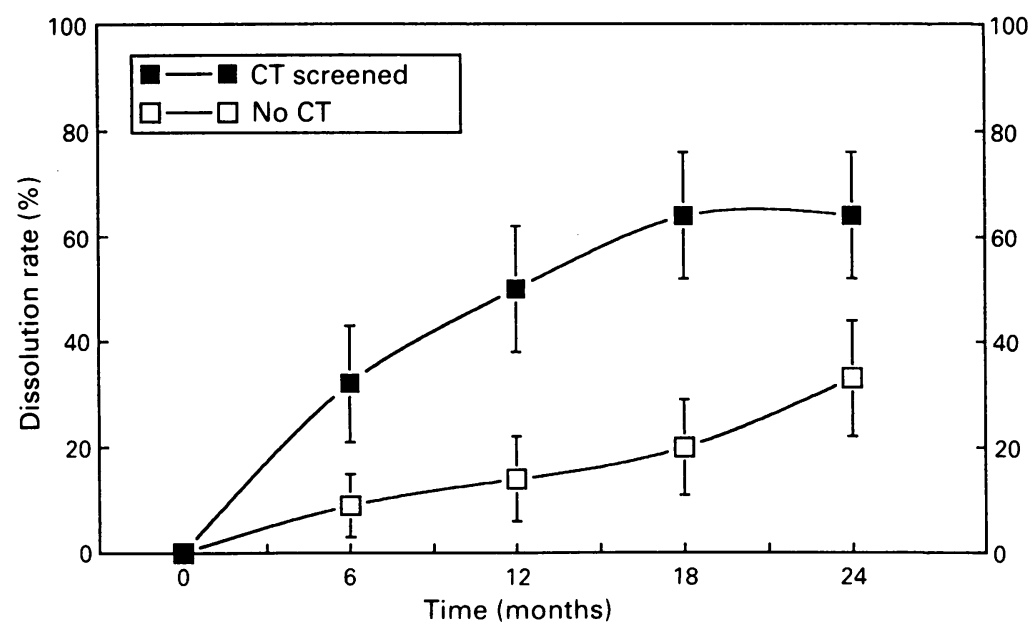

Figure 3: Complete gall stone dissolution rates (mean (SEM)) in the two subgroups of (i) 24 patients screened by computed tomography and (ii) 29 patients with no pretreatment computed tomography. stones in selected patients. Furthermore, they show that routine computed tomography before treatment, to exclude patients with calcified gall stones on the basis of a maximum stone attenuation value $>100 \mathrm{HU}$, significantly increases the probability complete stone dissolution.

The combination of CDCA and UDCA has been used in recent years by several groups ${ }^{10-16}$ in order to reduce the side effects of CDCA and the cost of UDCA while maintaining the efficacy of gall stone dissolution. Podda $e t a l^{15}$ have clearly shown in a prospective randomised trial that the combination of CDCA and UDCA results in better dissolution rates than UDCA alone.

UDCA and CDCA act somewhat differently to reduce biliary cholesterol saturation but, as our study shows, when combined at a dose of $5.0 \mathrm{mg}$ UDCA $/ \mathrm{kg} /$ day and $7.5 \mathrm{mg}$ CDCA $/ \mathrm{kg} /$ day the two bile acids produce a highly significant reduction in the mean biliary cholesterol saturation index from $1 \cdot 32$ to $0 \cdot 74$. This change in the saturation index is similar to that shown in other studies with the combination of the two bile acids ${ }^{1015} 16$ but does not take into account cholesterol present in biliary vesicles or in other non-micellar forms.

In the pooled group (computed tomography screened plus those not screened) of 55 patients, the complete dissolution rate after two years of UDCA plus CDCA treatment was only $44(8) \%$. However, this figure is biased by the outcome in the subgroup of patients screened by computed tomography. Had they been excluded, the two year complete dissolution rate in the patients without computed tomography would have been only $33(11) \%$ This is a lower figure than that obtained by others with the combination of UDCA and CDCA. For example, Podda $e t a l^{15}$ reported a complete dissolution rate of $60(6) \%$ at 24 months. However, their patients were highly selected in that almost half ( 29 of 60$)$ had small floating stones. Other investigators have shown lower mean dissolution rates of $25 \%^{12}$ and $27 \%^{16}$ after 24 months' treatment.

In our study, complete dissolution was defined rigorously by two normal ultrasound examinations. This approach is likely to result in lower but more accurate dissolution rates than those obtained using less stringent criteria. Thus, in another study from our unit in which UDCA alone was given in a dose of $8-10 \mathrm{mg} / \mathrm{kg} /$ day, complete dissolution occurred in $25-30 \%$ of patients when defined by oral cholecystography but in only $17-19 \%$ when defined by ultrasonography. ${ }^{4}$ This compares with an overall complete dissolution rate of $38 \%$ diagnosed by oral cholecystography in a third group of comparably selected patients from our unit who were treated with CDCA alone. ${ }^{2}$

In the present study, the combination of UDCA and CDCA was generally well tolerated. Although $25 \%$ of the patients reported some diarrhoea, it was usually mild and transient. This compares with an incidence of about $50 \%$ in patients given CDCA alone. ${ }^{2}$ Indeed, in CDCA treated patients, diarrhoea is a dose dependent side effect. ${ }^{53}$ Despite the fact that one quarter of the patients in the present study complained of diarrhoea, it necessitated withdrawal of treat- 
ment in only two, one of whom had pre-existing colonic pathology (diverticular disease).

Ultrasonography does not distinguish between radiolucent and radio-opaque stones, and because of our reliance on ultrasound (rather than oral cholecystography) to assess the response to treatment, we cannot be certain that surface gall stone calcification never developed in patients taking UDCA plus CDCA. However, since $46 \%$ of our patients achieved complete dissolution (as calculated in the overall group by LTA at 24 months), we can say that in these patients, at least, acquired gall stone calcification either did not occur or if it did, it was insufficient to prevent progress to complete dissolution. Furthermore, we do not know how many patients developed a non-functioning gall bladder, though from previous studies ${ }^{4}$ the incidence of this is less than $10 \%$ over two years. Reliance on ultrasonography may also affect the estimation of partial dissolution as there may be difficulty in detecting both the reduction in the size of small stones and the exact number of multiple stones.

Arguably, the most important single factor influencing the success of oral dissolution therapy is the selection of patients whose gall stones are likely to dissolve completely. Thus, stone size before treatment is known to affect the outcome of oral bile acid treatment: the smaller the stones, the better and more rapid the complete dissolution rates. Stones measuring $>15-20 \mathrm{~mm}$ in maximum diameter respond poorly or not at all. ${ }^{2}$ However, non-cholesterol stones or those of mixed composition containing appreciable amounts of calcium salts would not be expected to dissolve completely - even in bile rendered unsaturated in cholesterol by oral UDCA or CDCA, or both.

In the present study we assessed the impact of two methods of screening for non-cholesterol stones on dissolution efficacy: (i) measurement of biliary cholesterol saturation (a pretreatment saturation index of $>1.0$ usually $^{24}$ but not always, ${ }^{25}$ predicting non-cholesterol stones) and (ii) routine computed tomographic scanning of the gall bladder area before starting treatment. In the 21 patients who underwent bile lipid studies before treatment and completed one year's therapy, the differences in outcome between those with saturated bile and those with unsaturated bile did not reach statistica significance - possibly because of the small numbers of patients studied. Additionally, measurement of biliary cholesterol saturation indices ignores non-micellar solubilisation of cholesterol and although microscopy of the bile can enhance diagnostic accuracy, ${ }^{26}$ duodenal drainage is unpopular with patients and time consuming for the investigator. Outside clinical research protocols, therefore, it is seldom used today.

In contrast, computed tomography is well accepted by patients and although it is expensive, it involves minimal exposure to $x$ irradiation. As shown by the present results, it had a major impact on dissolution efficacy when used routinely to exclude patients with calcium containing stones that were judged unlikely to dissolve completely. Based on previous studies from our unit ${ }^{17}$ in which we related the maximum pretreatment gall stone HU score measured in vivo to gall stone composition and dissolvability - both measured in vitro in stones retrieved at cholecystectomy - in the present study, we chose a cut off point in maximum gall stone attenuation of $100 \mathrm{HU}$. This corresponds to a stone total calcium content of only $3 \%$ by weight, but even this small amount of calcium seems to be enough to impair complete dissolution with contact solvents such as $\mathrm{MTBE}^{27}$ However, measurement of gall stone attenuation by in vivo computed tomography is not precise ${ }^{28}$ and there is intrapatient, intramachine, and intermachine variability which we estimate to be approximately $\pm 10 \mathrm{HU}$ overall.

We believe that the observed improvement in dissolution efficacy as a result of routine computed tomographic screening before treatment, is due solely to the exclusion of patients with calcium containing stones. Thus, the patient and gall stone characteristics were comparable in patients screened and not screened by computed tomography. Furthermore, there was no bias as a result of including previously treated patients in the two treatment groups. In the present study, there were too few patients with recurrent stones to extend the results of previous studies from our own unit ${ }^{29}$ and elsewhere ${ }^{30}$ which suggest that most, but not all, recurrent stones are small, cholesterol rich, and easily dissolvable.

The present report is important not only because it extends the results of previous studies in which the combination of oral UDCA and CDCA has been used as the sole treatment for gall bladder stones, but also because $w^{31}$ and other $^{32-33}$ routinely use this combination of oral bile acids as adjuvant treatment after extracorporeal shock wave lithotripsy (ESWL). Although the aim of ESWL is to convert large stones into as small fragments as possible, ${ }^{33}{ }^{34}$ in only a minority of patients $(<20 \%)$ do the fragments pass spontaneously through the cystic duct: in the majority, the fragments must be dissolved. Thus, in a large multicentre doubleblind trial, patients treated with ESWL plus oral bile acids cleared their gall stones significantly better than those given ESWL plus placebo. ${ }^{35}$

It is possible to fragment calcium containing gall stones with ESWL. Since most of the resultant fragments remain within the gall bladder and must be dissolved, however, the results of lithotripsy plus oral bile acids in patients with calcified stones are appreciably worse than those in patients with radiolucent, cholesterol rich gall stones. ${ }^{32}$ For this reason, we have adopted a policy of screening with computed tomography all gall stone patients being considered for non-surgical treatment, and exclude from lithotripsy plus oral bile acids those who have maximum HU scores in excess of 100 . However, we have yet to establish that this approach is as effective in patients treated with the combination of ESWL plus oral bile acids as in those given UDCA plus CDCA alone. With both these treatments, the value of pretreatment computed tomography scanning could now be confirmed more rigorously in a prospective randomised trial.

We conclude that in appropriately selected 
patients gall stone dissolution with a combination of UDCA and CDCA can be remarkably successful and routine pretreatment computed tomography of the gall bladder seems to have an important role in improving this selection process by detecting, and thereby excluding from treatment, patients with non-cholesterol stones.

We thank Mr Younos Qureshi for technical assistance and Mrs Ann Hollington for preparation of the typescript. Thanks are also due to the St Martin's Group of Hospitals for partial financial support (JRFW and KAH). Parts of this study were presented at the British Society of Gastroenterology at the Autumn meetings in 1987 and 1989, and published as abstracts (Gut 1987; 28: A1360 and Gut 1989; 30: A1459).

I Schoenfield LJ, Lachin JM, et al. Chenodiol (chenodeoxycholic acid) for dissolution of gallstones: the National Cooperative Gallstone Study: a controlled trial of efficacy and safety. Ann Int med 1981; 95: 257-82.

2 Maton PN, Iser JH, Reuben A, Saxton HM, Murphy GM, Dowling RH. Outcome of chenodeoxycholic acid (CDCA) treatment in 125 patients with radiolucent gallstones. Medicine (Baltimore) 1982; 61: 86-97.

3 Bachrach WH, Hofmann AF. Ursodeoxycholic acid in the treatment of cholesterol cholelithiasis. Dig Dis Sci 1982; 27: treatment

4 Gleeson D, Ruppin DC, Saunders A, Murphy GM, Dowling RH. Final outcome of ursodeoxycholic acid treatment in 126 patients with radiolucent gallstones. Quart $\mathcal{F}$ Med 1990; 76: 711-29.

5 Mok HYI, Bell GD, Dowling RH. Effect of different doses of chenodeoxycholic acid on bile lipid composition and frequency of side effects in patients with gallstones. Lancet 1974; ii: $253-7$.

6 Albers JJ, Grundy SM, Cleary PA, et al. National cooperative gallstone study. The effect of chenodeoxycholic acid on lipoproteins and apoproteins. Gastroenterology 1982; 82: 638-46.

7 Maton PN, Murphy GM, Dowling RH. Ursodeoxycholic acid treatment of gallstones. Dose response study and possible mechanism of action Lancet 1977; ii: 1297-1301.

8 Von Bergman K, Epple-Gutsfeld M, Leiss O. Differences in the effects of chenodeoxycholic and ursodeoxycholic acid on biliary lipid secretion and bile acid synthesis in patients with gallstones. Gastroenterology 1984; 87: 136-43.

9 Roda E, Bazzoli F, Morselli Labate AM, et al. Ursodeoxycholic acid vs chenodeoxycholic acid as cholesterol gallstonedissolving agents: a comparative randomised study. Hepatology 1982; 2: 804-10

10 Podda M, Zuin M, Dioguardi ML, Festorazzi S, Dioguardi N. A combination of chenodeoxycholic acid and ursodeoxycholic acid is more effective than either alone in reducing biliary cholesterol saturation. Hepatology 1982; 2: 334-9.

11 Stiehl A, Raedsch R, Czygan P, Gotz R, Manner CH, Walker S. Effects of biliary bile acid composition on biliary cholesterol saturation in gallstone patients treated with
chenodeoxycholic acid and/or ursodeoxycholic acid. Gastrochenodeoxycholic acid and/or
enterology 1980; 79: 1192-8.

12 Roehrkasse R, Fromm H, Malavolti M, Tunuguntla AK, Ceryak S. Gallstone dissolution treatment with a combination of chenodeoxycholic and ursodeoxycholic acids: studies of safety, efficacy and effects on bile lithogenicity, bile acid pool, and serum lipids. Dig Dis Sci 1986; 31: 1032-40.

13 Czygan P. Efficacy of combined ursodeoxycholic and chenodeoxycholic acid treatment. In: Paumgartner G, Stiehl $\mathrm{A}$, Gerok W, eds. Bile acids and the liver. Lancaster: MTP Press, 1987: 343-4.

14 Thistle JL. Dissolution of gallstones using ursodeoxycholic acid with or without chenodeoxycholic acid or taurine. In: Paumgartner G, Stiehl A, Gerok W, eds. Bile acids and the liver. Lancaster: MTP Press, 1987: 353-4.
15 Podda M, Zuin M, Battezzati PM, Ghezzi C, de Fazio C Dioguardi ML. Efficacy and safety of a combination of chenodeoxycholic acid and ursodeoxycholic acid for gallstone dissolution: a comparison with ursodeoxycholic acid alone. Gastroenterology 1989; 96: 222-9.

16 Janowitz P, Swobodnik W, Wechsler JG, Fischer S, Tudyka J Ditschuneit $\mathrm{H}$. Results of an index-controlled combination therapy with urso- and chenodeoxycholic acid. In Swobodnik W, Ditschuneit H, Soloway RD, eds. Gallstone disease: pathophysiology and therapeutic approaches. Heidelberg: Springer, 1990: 135-45.

17 Rajagopal S, Bills P, Keightley A, Murphy GM, Dowling RH. Predictive value of computed tomography (CT) scanning of the gall bladder in determining gall stone type. Gut 1988;29: A1487.

18 Baron RL, Rohrmann CA Jr, Lee SP, Shuman WP, Teefey SA. CT evaluation of gallstones in vitro: correlation with SA. CT evaluation of gallstones in vitro: correlation
chemical analysis. Am $\mathcal{F}$ Radiol $1988 ; 151$ : 1123-28.

19 Janowitz P, Zöller A, Swobodnik W, Wechsler JG, Schumacher KA, Ditschuneit H. Computed tomography evaluation of radiolucent gallstones in vivo. Gastrointes Radiol 1990; 15: 58-60.

20 Dowling RH. Medical treatment of gallbladder stones: good news and bad news. In: Paumgartner G, Stiehl A, Gerok W, eds. Trends in bile acid research. Dordrecht/Boston/London: Kluwer Academic Publishers, 1989: 283-305.

21 Armitage P, Berry G. Statistical methods in medical research, 2nd ed. Oxford: Blackwell, 1987.

22 Howard PJ, Gleeson D, Murphy GM, Dowling RH. Ursocholic acid: bile acid and bile lipid dose response and clinical acid: bile acid and bile lipid dose response and clinical

23 Iser JH, Dowling RH, Mok HYI, Bell GD. Chenodeoxycholi acid treatment of gallstones - a follow-up report and analysis of factors influencing response to therapy. $N \mathrm{Engl} F \mathrm{~F}$ Med 1975; 293: 378-83.

24 Dolgrin SM, Schwartz JS, Kressel HY, et al. Identification of patients with cholesterol or pigment gallstones by discriminant analysis of radiographic features. $N \mathrm{Engl} \mathcal{F} \mathrm{Med}$ 1981; 304: 808-11.

25 Dowling RH, Murphy GM, Iser J. Dissolution of gallstones by chenodeoxycholic acid. In: Taylor W, ed. The hepatobiliary system: fundamental and pathological mechanisms. New York: Plenum, 1976; 485-501.

26 Ros E Navarro S, Fernández I, Reixach M, Ribó JM, Rodés J. Utility of biliary microscopy for the prediction of the chemical composition of gallstones and the outcome of dissolution dissolution therapy with
entrology $1986 ; 91 ; 703-12$.

27 Rajagopal SU, Keightley A, Bills P, Walters JRF, Murphy GM, Dowling RH. Predictive value of pre-treatment C' scanning vs conventional radiology in determinin composition, dissolvability and fragmentability of gallbladder stones. F Hepatol 1989; 9: S211.

28 LeviC, Gray E, McCullough EC, Hattery RR. The unreliability of CT numbers as absolute values. Am f Radiol 1982; 139. 443-7.

29 Ruppin DC, Dowling RH. Is recurrence inevitable after gallstone dissolution by bile-acid treatment? Lancet 1982; $181-5$.

30 O'Donnell LDJ, Heaton KW. Recurrence and re-recurrence of gallstones after medical dissolution: a longterm follow up. Gut 1988; 29: 655-8.

31 Hood KA, Keightley A, Dowling RH, Dick JA, Mallinson $\mathrm{CN}$. Piezo-ceramic lithotripsy of gallbladder stones: initia experience in 38 patients. Lancet 1988; i: 1322-4.

32 Sackmann M, Delius M, Sauerbruch T, et al. Shock-wave lithotripsy of gallbladder stones. The first 175 patients. $N$ Englf Med 1988; 318: 393-7.

33 Ponchon T, Barkun AN, Pujol B, Mestas JL, Lambert R. Gallstone disappearance after extracorporeal lithotripsy and oral bile acid dissolution. Gastroenterology 1989; 97: 457-63.

34 Podda M, Zuin M, Dowling RH, Barbara L, Fromm H, Salen G. Extracorporeal lithotripsy of gall-bladder stones: results, cost-effectiveness, future perspectives. Gastroenterol Int 1989; 2: 107-110.

35 Schoenfield LJ, Berci G, Carnovale RL, et al. The effect of ursodiol on the efficacy and safety of extracorporeal shockwave lithotripsy of gallstones: The Dornier National Biliary Lithotripsy Study. N Engl I Med 1990; 323: 1239-45. 